\title{
Microcirculation in cardiogenic shock: from scientific bystander to therapy target
}

\author{
Christian Jung*, Alexander Lauten and Markus Ferrari \\ See related research by Munsterman et al., http://ccforum.com/content/14/4/R161
}

\begin{abstract}
Despite diagnostic and therapeutic improvements, mortality rates in patients with cardiogenic shock remain relatively high. Several studies showed that cardiogenic shock is associated with alterations in the microvascular circulation. These alterations may be reversed by extracorporeal support devices. A study by Munsterman and colleagues adds to the body of evidence showing that in patients deemed ready for discontinuing intra-aortic balloon pump (IABP) support, microcirculatory flow in small vessels increases after ceasing IABP therapy. This study not only highlights the need for optimal timing of weaning from IABP support but also supports recent findings that global hemodynamics do not necessarily result in changes of microvascular perfusion. All modalities of modern treatment in cardiogenic shock need to be evaluated for their effect on the microcirculation. Microcirculatory evaluations should be part of randomized controlled trial protocols. More effort is needed to improve outcomes and understand the microcirculation as a therapy target and not as a silent bystander.
\end{abstract}

Despite diagnostic and therapeutic improvements, cardiogenic shock (CS) is still the most common cause of death in patients with acute myocardial infarction. Although percutaneous coronary intervention (PCI), inotropes, fluids, adjunctive medication, intra-aortic balloon pump (IABP), and ventricular assist devices are widely available, mortality rates in patients with CS remain high (40\% to 50\%). Therefore, Munsterman and

*Correspondence: christian.jung@med.uni-jena.de

First Department of Internal Medicine, (Cardiology, Angiology, Pneumology, Intensive Care Medicine), Friedrich-Schiller University, Erlanger Allee 101, 07740 Jena, Germany colleagues [1], whose investigation of the microcirculation in patients with IABP was reported in the previous issue of Critical Care, have addressed an important topic.

In the last five years, an important body of knowledge showing the pathophysiological importance of the microcirculation in the development of multiple organ failure in critically ill patients has been built up. This has been made possible because of the development of novel techniques to either directly visualize or indirectly evaluate microvascular perfusion [2]. In CS, microvascular alterations have been observed, resulting in a decrease of vessel density, the proportion of perfused capillaries, or microvascular flow [3,4]. Different treatment strategies, including pharmacological interventions and mechanical assist devices, may lead to microcirculatory improvement in CS [5-7].

In their article, Munsterman and colleagues show that in patients deemed ready for discontinuing IABP support, microcirculatory flow in small vessels increases after ceasing IABP therapy. The authors conclude that IABP impairs microvascular perfusion in hemodynamically recovered patients. These important findings not only highlight the need for optimal timing of weaning from IABP support but also demonstrate that global hemodynamics do not necessarily result in changes of microvascular perfusion $[8,9]$. In theory, IABP improves peak diastolic pressure and this may also translate into better microcirculation. Although sufficient cardiac output and arterial pressure are prerequisites for adequate microvascular perfusion, their relationship is very complex. Changes in microvascular vessel density and flow, their heterogeneity, autoregulation, and response to therapeutic interventions might be dissociated from systemic effects $[2,9]$. Furthermore, with the knowledge of the study by Munsterman and colleagues, it is obviously important to realize that, depending on the stage of the disease course, an intervention has different effects on the microcirculation.

Modern therapy of CS consists of an adequate regime with revascularization, drug treatment, and mechanical support [10]. Although there is no question about the 


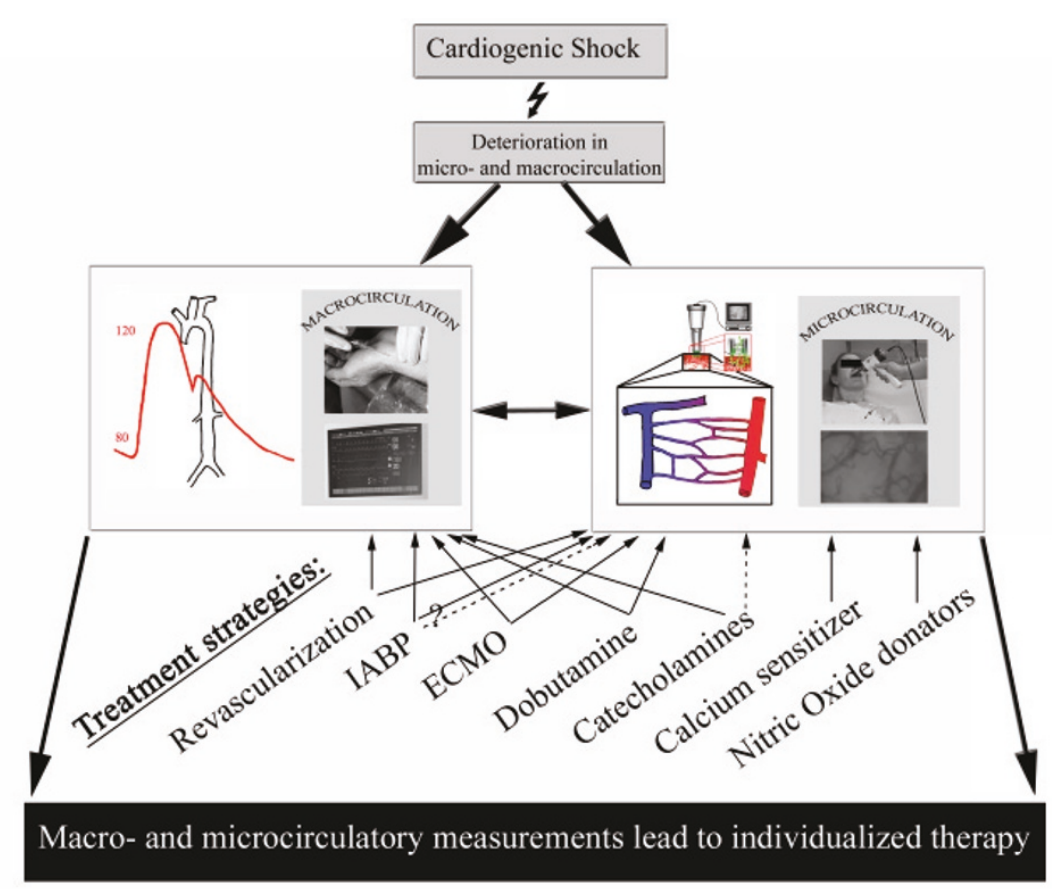

Figure 1. Treatment strategies affecting macrovascular and microvascular perfusion in cardiogenic shock. Effect of treatment strategies on microcirculation: solid arrow, positive effect; dotted arrow, negative effect. ECMO, extracorporeal membrane oxygenation; IABP, intra-aortic balloon pump.

survival advantage of early revascularization and its beneficial consequences for the macrocirculation, there is no systematic study on its effects on microcirculation. However, revascularization reverses the spiral of progressive left ventricular dysfunction and therefore is understood to improve the microcirculation.

Different drugs, particularly fluids, vasopressors, inotropes, levosimendan, and vasodilators, influence the hemodynamics of patients with CS. The administration of fluids in CS is based mainly on pathophysiological considerations and has not been studied in adequate randomized clinical trials. No data regarding fluid administration and microcirculatory changes in the setting of CS are available. Similar to fluid administration, the choice of vasopressor and inotropic therapy is based mainly on individual experience and institutional policy; furthermore, this choice differs between guidelines. However, vasopressors might be able to stabilize the mean arterial pressure, but their use has negative consequences for the perfusion within microvasculature [11]. In small observatory studies, beneficial effects on microcirculatory indices in CS have been described for levosimendan and nitroglycerin $[5,12]$.

Owing to the lack of evidence for the use of IABP in CS after successful revascularisation of occluded infarct vessels, its role remains controversial. To date, there is no adequately powered randomized clinical trial available, and knowledge is based on observational trials or trials before the era of primary PCI. IABP support improves microvascular flow in unstable patients; however, different time points of the disease seem to be of importance $[6,9]$.

Figure 1 summarizes therapeutic strategies affecting microvascular perfusion in CS. All interventions should be evaluated for their effect at the microcirculatory level. Microcirculatory evaluations need to be part of randomized controlled trial protocols. Guiding inter-individual modern therapy of CS with in vivo visualization tools may allow a more specific and appropriate therapy regime and improve outcomes. More effort is needed to understand the microcirculation as a therapy target and not as a silent bystander.

\section{Abbreviations}

CS, cardiogenic shock; IABP, intra-aortic balloon pump; $\mathrm{PCl}$, percutaneous coronary intervention.

\section{Competing interests}

The authors declare that they have no competing interests.

Published: 6 September 2010

\section{References}

1. Munsterman LDH, Elbers PW, Ozdemir A, van Dongen EP, Van Iterson M, Ince C: Withdrawing intra-aortic balloon pump support paradoxically improves microvascular flow. Crit Care 2010, 14:R161.

2. De Backer D, Ospina-Tascon G, Salgado D, Favory R, Creteur J, Vincent JL: Monitoring the microcirculation in the critically ill patient: current methods and future approaches. Intensive Care Med 2010 Aug 6. [Epub ahead of print]. 
3. De Backer D, Creteur J, Dubois M-J, Sakr Y, Vincent J-L: Microvascular alterations in patients with acute severe heart failure and cardiogenic shock. Am Heart J 2004, 147:91-99.

4. Jung C, Ferrari M, Roediger C, Fritzenwanger M, Goebel B, Lauten A, Pfeifer R, Figulla HR: Evaluation of the sublingual microcirculation in cardiogenic shock. Clin Hemorheol Microcirc 2009, 42:141-148.

5. den Uil CA, Lagrand WK, Spronk PE, van der Ent M, Jewbali LS, Brugts JJ, Ince C, Simoons ML: Low-dose nitroglycerin improves microcirculation in hospitalized patients with acute heart failure. Eur J Heart Fail 2009, 11:386-390

6. Jung C, Rodiger C, Fritzenwanger M, Schumm J, Lauten A, Figulla HR, Ferrari M: Acute microflow changes after stop and restart of intra-aortic balloon pump in cardiogenic shock. Clin Res Cardio/ 2009, 98:469-475.

7. Jung C, Ferrari M, Gradinger R, Fritzenwanger M, Pfeifer R, Schlosser M, Poerner TC, Brehm BR, Figulla HR: Evaluation of the microcirculation during extracorporeal membrane-oxygenation. Clin Hemorheol Microcirc 2008, 40:311-314

8. den Uil CA, Klijn E, Lagrand WK, Brugts JJ, Ince C, Spronk PE, Simoons ML:
The microcirculation in health and critical disease. Prog Cardiovasc Dis 2008, 51:161-170.

9. De Backer D, Ortiz JA, Salgado D: Coupling microcirculation to systemic hemodynamics. Curr Opin Crit Care 2010, 16:250-254.

10. Thiele H, Allam B, Chatellier G, Schuler G, Lafont A: Shock in acute myocardial infarction: the Cape Horn for trials? Eur Heart J 2010, 31:1828-1835

11. Fries M, Weil MH, Chang YT, Castillo C, Tang W: Microcirculation during cardiac arrest and resuscitation. Crit Care Med 2006, 34:\$454-457.

12. Wimmer $R$, Janusch $M$, Lemm H, Winkler M, Buerke M, Werdan K: Effect of levosimendan on microcirculation in cardiogenic shock [in German]. Intensivmed 2007, 44:227-261.

\section{doi:10.1186/cc9244}

Cite this article as: Jung C, et al:. Microcirculation in cardiogenic shock

from scientific bystander to therapy target. Critical Care 2010, 14:193. 